\title{
Research on constraint for conjunction between beam and shear wall
}

\author{
X.M. Chen ${ }^{\text {a }}$ J. Duan, Y.G. Li \\ China state construction technical center, Beijing, China \\ aHanee@126.com
}

\begin{abstract}
Keywords: Beam element, Shell element, Constraint.
Abstract. Different with definition of continuum mechanics used for beam element, in-plane drilling degrees of shell element are usually based on Allman mode or additional rigid rotation of element sides, and assumption of plane section for beam element is another inconsistency with shell element, at last, the beam section may cover more than one node of shell element with mesh refinement. Therefore, direct connection for these two degrees of freedom may be inappropriate, special connector elements or constraints should be introduced to enforce the stiffness of connection. Numerical results show that the influence of constraints on periods can be neglected, but bending moment at beam end should be simulated with proper additional constraint, compared with the other modes, constraint on drilling degrees of freedom is much more effective.
\end{abstract}

\section{Introduction}

In structural analysis, to simulate stiffness accurately depends on reasonable compatibility between elements. Except the fundamental form of nodal deforming compatibility, there are some special compatible controls need be simulated with constraint, such as the constraint for conjunction between beam and shear wall which is used widely in professional structural software.

Frameworks are simulated with beam elements, and the drilling degrees of freedom are defined with continuum mechanics mode. Otherwise, shear walls and slabs are simulated with shell elements, but for shell elements, the relationship between nodal rotation $\omega$ according to continuum mechanics and drilling degrees of freedom $\theta$ in plane elements has not been formulated clearly, so Allman method[1] and additional rigid rotation of element sides[2] are recommended to be used instead of continuum mechanics mode.

Thus, the definitions of drilling degrees of freedom for beam and in-plane of shell are usually inconsistent. On the other hand, plane-section assumption is used for development of most beam elements, this may cause incompatible conforming at the conjunction between beam section and sides of shell element, and the stiffness of connection would be underestimated. At last, for some beams, the section of beam may contain more than one nodes of shell element, therefore, the stiffness would be also underestimated if only one node was modeled to resistant the bending of beam element. For these reasons, when a beam was supported with shear wall, which was parallel with the beam, professional software for structural analysis would add penalty elements at the corner of shear walls by default $[3,4,5]$ to enforce the stiffness of conjunction. With this method, the bending moment of according beam end would be increased, and reinforcement would be more reasonable.

Various constraints or special connector elements are available in general FEA software, but it wouldn't be applied by software automatically. For analysis of high buildings, this kind of constraint was often neglected by most of engineers because of complexity of modeling process and puzzle of over-constraint.

With different constraints, results of structural period and bending moments of beam end are researched by using numerical method. Numerical results show that the influence of constraints on structural periods can be neglected, but engineers should pay more attention to the variation of bending moments at beam end induced by constraints. As rigid constraint may amplify the connection stiffness improperly, and constraint for drilling degree of freedom can present similar results as penalty method, then the later is recommended for enforcing the conjunction between beam elements and shell elements in structural analysis. 


\section{Introduction of constraint}

Penalty method is one kind of multi-point constraint used widely in finite element method. The constraint equation can be introduced into the energy functional by penalty factor $\alpha$ as follows:

$$
\Pi^{*}=\Pi+\frac{1}{2} \alpha C^{T} C
$$

In this method, the selection of penalty factor $\alpha$ may be difficult. Usually, a big number of $\alpha$ could present better constraint effect, but the negative effect is ill-conditioned equations. So experiences are necessary for this method.

In software for structural design, penalty elements are used to enforce constraints at the conjunction between beams and shear walls[6]. By using this kind of special element, more reasonable bending moment at beam end would be presented, and as the result, the reinforcement for this bending moment is also increased to improve the integrity.

Equal Constraint in SAP[7] and Universal Constraint in ABAQUS[8] can be used equivalently.

An Equal Constraint causes all of its constrained joints to move together with the same displacements for each selected degree of freedom, taken in the constraint local coordinate system. The other degrees of freedom are unaffected. This kind of constraint differs from the rigid-body types of constraints in that there is no coupling between the rotations and the translations.

Connection type Universal in ABAQUS provides a connection between two nodes where the rotations are fixed about one local direction and free about two others.

For both Equal Constraint and Universal Constraint, with the definition of local coordinate system, the rotations of nodes about local axis $\mathrm{e}_{2}^{\mathrm{a}}$ and $\mathrm{e}_{2}^{\mathrm{b}}$, which are in the height of beam section, would be constrained to be equal as shown in Fig.1.
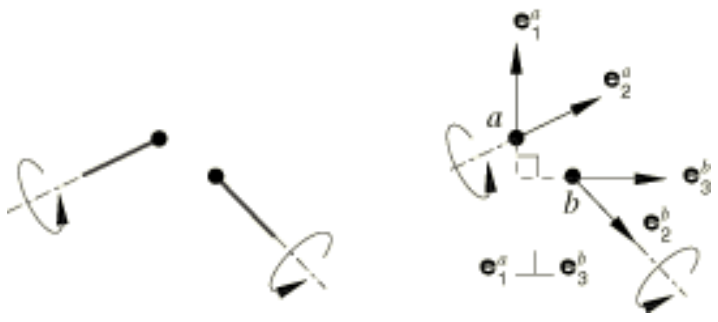

Fig. 1 Equal or Universal

A Body Constraint in SAP causes all of its constrained joints to move together as a three-dimensional rigid body. By default, all degrees of freedom at each connected joint participate. However, a subset of the degrees of freedom can be selected to be constrained.

The similar constraint is named as Beam in ABAQUS, this kind of connection provides a rigid beam connection between two nodes.

This kind of constraint can be used to:

- Model rigid connections, such as where several beams and/or columns frame together

- Connect together different parts of the structural model that were defined using separate meshes

- Connect Frame elements that are acting as eccentric stiffeners to Shell elements

By this connection, all the six components are constrained.

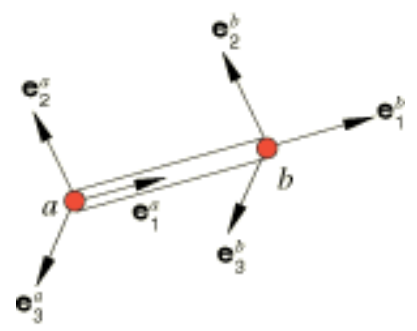

Fig.2 Body or Beam 


\section{Numerical example}
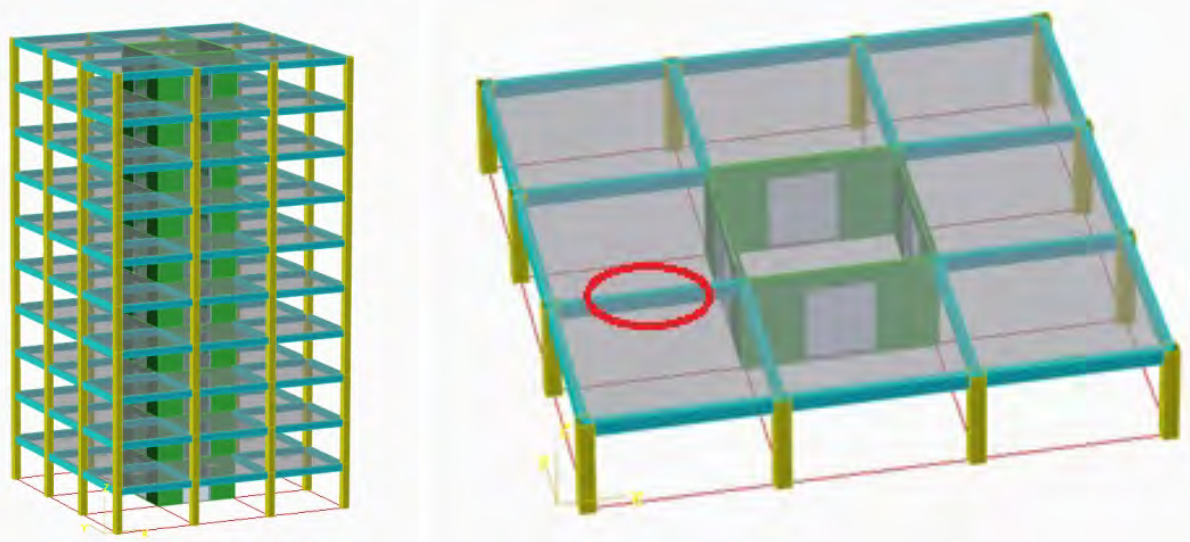

Fig.3 Model of numerical example

A ten-floor frame-core tube structure shown in Fig.3 is taken as example for numerical analysis. The beam marked in Fig.3(b) are researched for reasonable mechanical model. Four kinds of constraints are used for simulating the conjunction of beam and shear wall, include panel method in YJK, Equal/Universal Constraint in SAP/ABAQUS, Body/Beam constraint in SAP/ABAQUS, and none constraint in SAP and ABAQUS. Results of periods and bending moment are presented for different constraints.

\section{Numerical results}

(1) Periods result

The periods of different models are listed in Fig.1.

As professional software for concrete structures, the mass is accumulated to the top of each floor in YJK, therefore, its periods are longer than others. For SAP and ABAQUS, the deviation of periods induced by different constraint can be neglected.

Fig.1 Periods of different models

\begin{tabular}{|c|c|c|c|c|c|c|c|}
\hline Software & YJK & \multicolumn{3}{|c|}{ SAP } & \multicolumn{3}{c|}{ ABAQUS } \\
\hline Constraint & Panel & None & Equal & Body & None & Universal & Beam \\
\hline T1 & 1.12 & 1.01 & 1.01 & 1.01 & 1.01 & 1.00 & 1.00 \\
\hline T2 & 1.02 & 0.98 & 0.97 & 0.97 & 0.98 & 0.97 & 0.96 \\
\hline T3 & 1.02 & 0.98 & 0.97 & 0.97 & 0.98 & 0.97 & 0.96 \\
\hline
\end{tabular}

(2) Bending moments of beams

Bending moments of the beam in 1st floor and the beam in 10st floor are shown in Fig.4 and Fig.5 respectively.

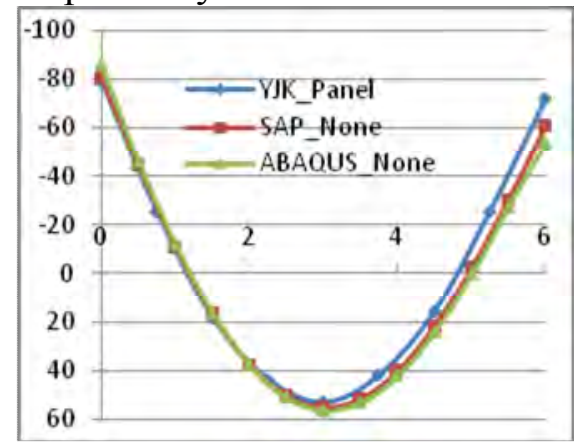

(a) Panel/None/None

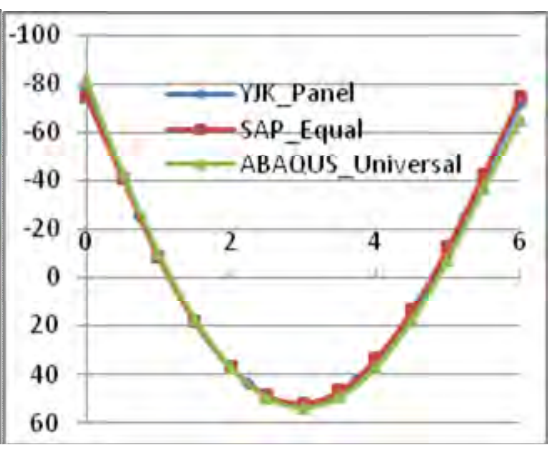

(b) Panel/Equal/Universal

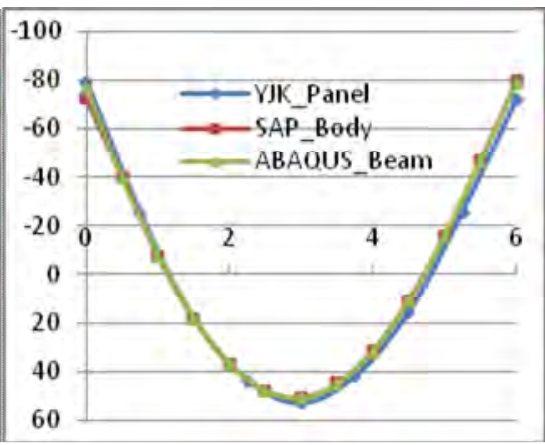

(c) Panel/Body/Beam

Fig.4 Bending moments of beam in 1st Floor 


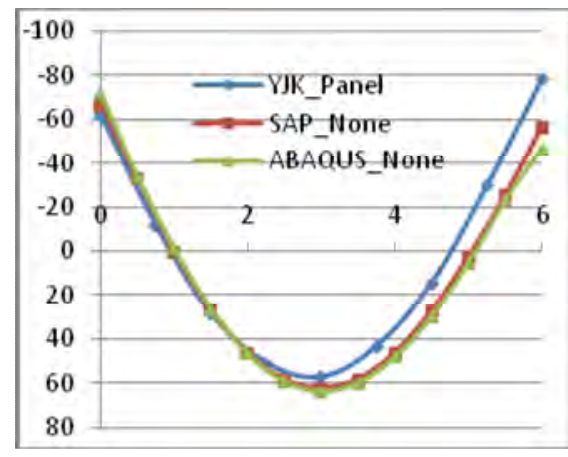

(a) Panel/None/None

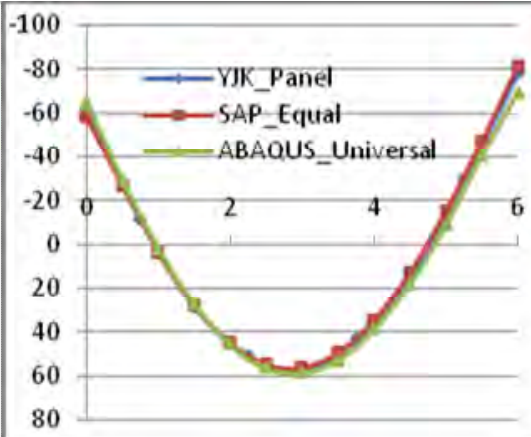

(b) Panel/Equal/Universal

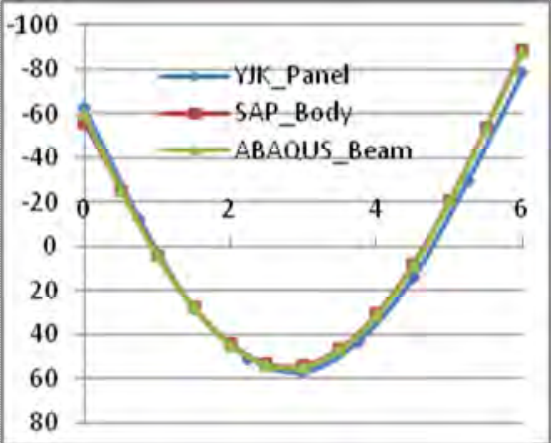

(c) Panel/Body/Beam

Fig. 5 Bending moments of beam in 10 st floor

As shown in Fig.4 and Fig.5, bending moments at right end are influenced significantly by different constraint s especially for the beam in 10st floor. In fact, the beam was connected to shear wall within the height of its section but not only the corner of shear wall, and what's more, the section of beam is always assumed to be a plane under deflection. By using different constraints, more than one node in shear wall can contribute stiffness for bending resistant of beam. Body Constraint in SAP and Beam Constraint in ABAQUS can satisfy the assumption of plane section completely which is only an idealized condition. Unlike rigid constraint, Panel method, Equal Constraint in SAP and Universal Constraint in ABAQUS can contribute elastic stiffness for this assumption.

Results of different constraints for SAP and ABAQUS are also shown in Fig.5 and Fig.6 respectively.

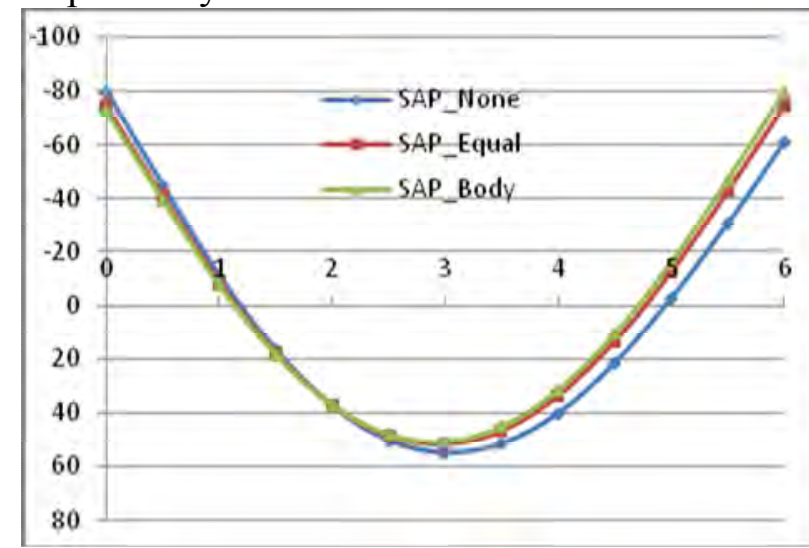

(a) Bending moments of beam in $1^{\text {st }}$ Floor

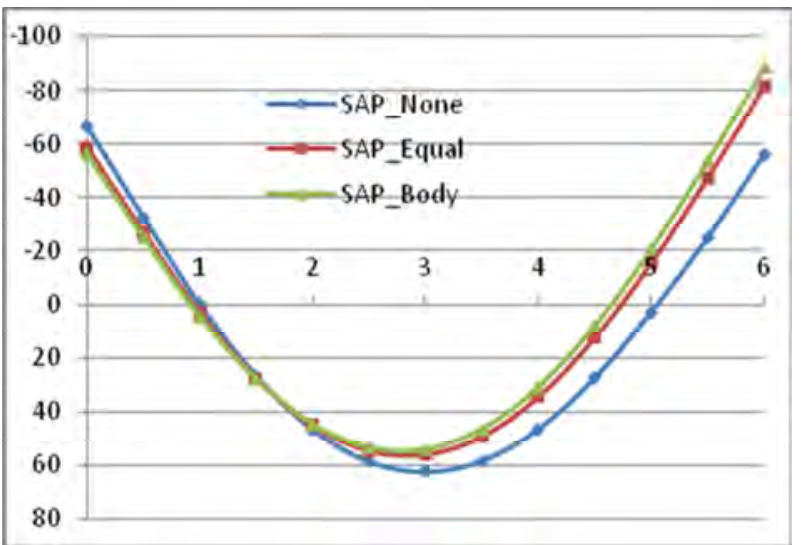

(b)Bending moments of beam in $10^{\text {st }}$ Floor

Fig. 6 Comparison of SAP results

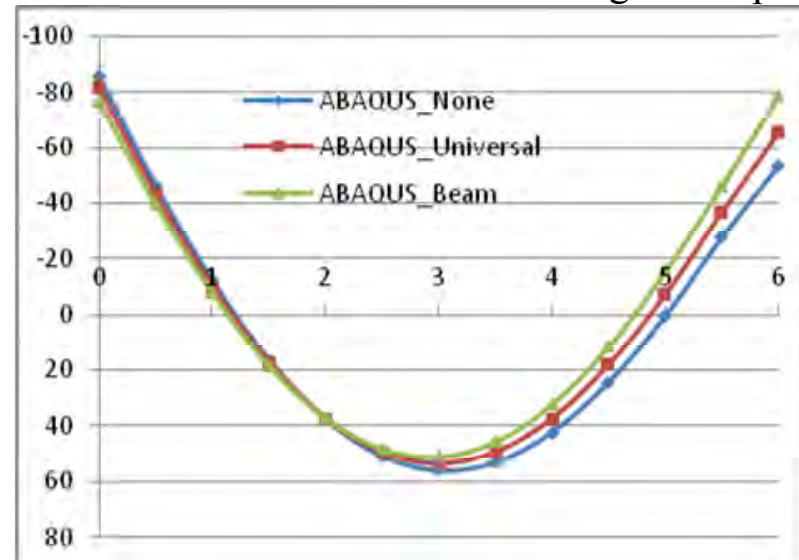

(a) Bending moment of beam in 1st Floor

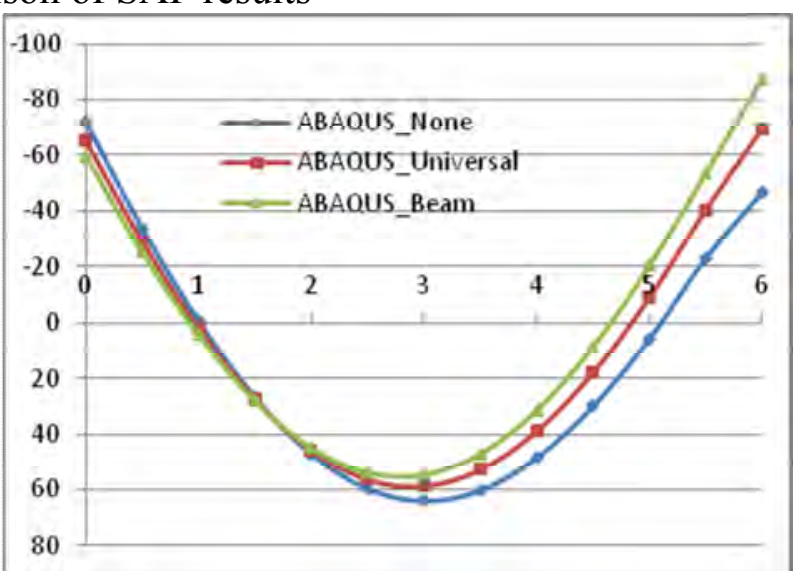

(b)Bending moment of beam in 10st Floor

Fig.7 Comparison of ABAQUS results

The results in Fig. 6 and Fig.7 show that the bending moments at right end are quite different under different constraints. The deviation in 10st is much more significant than 1st floor because of the difference of vertical deformation. As the assumption for Body Constraint of SAP and Beam 
Constraint in ABAQUS are too rigid, usually, constraint on drilling degree of freedom by using Equal of SAP of Universal of ABAQUS can present satisfied solution.

\section{Conclusions}

Frameworks and shear walls in high buildings are simulated with different finite elements. Compared with beam element, the in-plane drilling degrees of shell element for shear walls are based on different physical meanings. On the other hand, the assumption of plane section in beam element should also be taken into account, otherwise, the deformation of beam section could not keep compatible with the sides of shell element. At last, with mesh refinement, the beam section may cover more than one node of shell element. Therefore, direct connection for these two kinds of degree of freedom may be inappropriate, and special connector elements or constraints should be introduced to enforce the stiffness of connection. Numerical results show that the bending moment of beam end would be underestimated without any constraints, on the contrary, it would be overestimated with rigid constraints, and constraint for drilling degrees of freedom can present more reasonable results.

\section{References}

[1] Allman D J. A quadrilateral finite element including vertex rotation for plane elasticity analysis. Int J Numer Meth Eng, 1988, 26:717

[2] Yu-Qiu Long, Song Cen, Zhi-Fei Long. Advanced Finite Element Method in Structural Engineering. Springer-Verlag GmbH Berlin Heidelberg \& Tsinghua University Press, Beijing, 2009

[3] PKPM Inc(2009). PMSAP User Manual(In Chinese).

[4] GSCAD building software Inc(2010). GSSAP User Manual(In Chinese).

[5] YJK Inc(2012). YJK User Manual, V1.7(In Chinese).

[6] Huang Jifeng, Du Wenbo, Shao Hong, et. al. Super element of shear wall with generalized compatibility. Chinese Quarterly of Mechanics. 2008, 29(1):150

[7] CSI Inc(2010). SAP2000 User Manual, V14.2.4.

[8] ABAQUS Inc(2006). ABAQUS User Manual, V6.5.5. 